Instituto Internacional de Investigación y Desarrollo Tecnológico Educativo INDTEC, C.A.

DOI: https://doi.org/10.29394/Scientific.issn.2542-2987.2021.6.22.21.397-417

OAI-PMH: http://www.indteca.com/ojs/index.php/Revista Scientific/oai

Ensayo Original / Original Essay

\title{
Las aplicaciones web, fuente de soluciones y exclusión para la
} educación en tiempos de COVID-19

\author{
Autores: Manuel Esteban Rojas Bustos \\ Universidad Nacional de Educación, UNAE \\ esteban.rojasb7845@hotmail.com \\ Azogues, Ecuador \\ https://orcid.org/0000-0002-8350-0240 \\ Lissbeth Johanna Aucancela Coraizaca \\ Universidad Nacional de Educación, UNAE \\ liss.joha05@gmail.com \\ Azogues, Ecuador \\ https://orcid.org/0000-0002-5889-5850
}

\section{Resumen}

El uso de las Tecnologías de la Información y la Comunicación (Tics) y el gran conjunto de aplicaciones web que la conforman han sido esenciales para la continuidad del proceso educativo, paralizado por la pandemia de la COVID-19, generando que docentes, autoridades educativas y estudiantes se vean obligados a no asistir a los centros educativos. Teniendo como principal solución a esta problemática, continuar el proceso educativo, por la modalidad virtual, en donde las Tics han sido la principal solución, sin embargo, la utilización de estas ha ocasionado varios problemas para estudiantes y docentes. Es por ello que, el presente trabajo se basó en una investigación bibliográfica, tomando como referente a autores como Martínez (2018); y Enríquez (2020); entre otros, siendo así que, el objetivo de esta investigación es dar un breve análisis de las aportaciones que han brindado las TIC y el conjunto de diversas aplicaciones web que la conforman, y al mismo tiempo demostrar la exclusión que se ha generado, por su mal uso o por falta de acceso a las mismas, en el nivel de educación superior ecuatoriano, teniendo como conclusión principal que, las TIC deben ser implementadas como un acceso universal, para que no se produzcan etiquetajes o clasificaciones en el ámbito educativo.

Palabras claves: tics; aplicaciones; web; exclusión.

Código de clasificación internacional: 5801.99 - Otras (Educación y TIC).

\section{Cómo citar este ensayo:}

Rojas, M., \& Aucancela, L. (2021). Las aplicaciones web, fuente de soluciones y exclusión para la educación en tiempos de COVID-19. Revista Scientific, 6(22), 397-417, e-ISSN: 2542-2987. Recuperado de: https://doi.org/10.29394/Scientific.issn.2542-2987.2021.6.22.21.397-417

Fecha de Recepción: 15-07-2021
Fecha de Aceptación: 09-10-2021
Fecha de Publicación: 05-11-2021 
Instituto Internacional de Investigación y Desarrollo Tecnológico Educativo INDTEC, C.A.

DOI: https://doi.org/10.29394/Scientific.issn.2542-2987.2021.6.22.21.397-417

OAI-PMH: http://www.indteca.com/ojs/index.php/Revista Scientific/oai

Ensayo Original / Original Essay

\title{
Web applications, source of solutions and exclusion for education in times of COVID-19
}

\begin{abstract}
The use of Information and Communication Technologies (ICTs) and the great set of web applications that make it up have been essential for the continuity of the educational process, paralyzed by the COVID-19 pandemic, generating that teachers, educational authorities and students are forced not to attend educational centers. Having as the main solution to this problem, continue the educational process, through the virtual modality, where ICTs have been the main solution, however, the use of these has caused several problems for students and teachers. That is why the present work was based on a bibliographic research, taking as a reference authors such as Martínez (2018); and Enríquez (2020); among others, since the objective of this research is to give a brief analysis of the contributions that ICT and the set of various web applications that make it up have provided, and at the same time demonstrate the exclusion that has been generated, due to their misuse or lack of access to them, at the Ecuadorian higher education level, having as a main conclusion that ICT should be implemented as universal access, so that no labeling or classifications are produced in the educational field.
\end{abstract}

Keywords: tics; applications; web; exclusion. International classification code: 5801.99 - Other (Education and ICT).

\footnotetext{
How to cite this essay:

Rojas, M., \& Aucancela, L. (2021). Web applications, source of solutions and exclusion for education in times of COVID-19. Revista Scientific, 6(22), 397-417, e-ISSN: 2542-2987. Recovered from: https://doi.org/10.29394/Scientific.issn.2542-2987.2021.6.22.21.397-417
}

Date Received: 15-07-2021
Date Acceptance: 09-10-2021
Date Publication: 05-11-2021 


\section{Introducción}

El COVID-19 se extendió por todo el mundo de manera veloz, que no dio tiempo para poder establecer soluciones o planes de contingencia que ayudaran a disminuir los contagios, declarándose por la Organización Mundial de la Salud (OMS, 2020): el 11 de marzo, como una pandemia, que llego a la mayoría de países del mundo, entre unos de ellos Ecuador, obligando al gobierno nacional a declarar estado de emergencia sanitaria el 12 de marzo, y posteriormente estado de excepción a nivel nacional, cerrando así la mayoría de campos laborales, entre ellos el sector educativo.

Por consiguiente, género que se suspendan las clases como una estrategia para frenar los contagios, siendo así que el Ministerio de Educación (MINEDUC, 2020): "mediante Acuerdo Ministerial No. MINEDUC-MINEDUC2020-00014-A de 15 de marzo de 2020, la señora Ministra de Educación dispuso la suspensión de clases en todo el territorio nacional" (pág. 2); suspendiendo las mismas por un periodo de cuarenta días, para posteriormente volver a clases bajo una nueva modalidad, siendo esta, la educación virtual, teniendo que pasar drásticamente de recibir clases en un aula física, para recibirlas en el hogar, mediante un dispositivo electrónico que tenga una conexión estable a internet, o también por medio de radios o televisores, demostrando que la tecnología actual o las conocidas TIC, son la principal fuente de solución para poder continuar con el proceso educativo en el contexto ecuatoriano en tiempos de pandemia.

En este sentido, las tecnologías de la información o comunicación, desde su aparición han sido de gran beneficio para la humanidad, sobre todo al momento de aplicarlas en el campo educativo, ya que, han permitido que se desarrollen habilidades en los estudiantes, que tal vez en las aulas físicas nunca se podrán conseguir. También, ha permitido que los educandos puedan trabajar de forma colaborativa, y que su proceso de enseñanza-aprendizaje, como lo manifiesta Martínez (2018): “[...] se haga menos tedioso, más rápido 
Instituto Internacional de Investigación y Desarrollo Tecnológico Educativo INDTEC, C.A.

DOI: https://doi.org/10.29394/Scientific.issn.2542-2987.2021.6.22.21.397-417

OAI-PMH: http://www.indteca.com/ojs/index.php/Revista Scientific/oai

Ensayo Original / Original Essay

y motivante [...]" (pág. 156); puesto que, tienen a su disponibilidad un gran contingente de herramientas tecnológicas que permiten buscar información acorde a las necesidades de cada uno de ellos.

Además, otro de los grandes beneficios que ha generado las Tecnologías de la Información y la Comunicación a la humanidad es la comunicación, lo cual ha sido puesto en evidencia en la época actual, en donde el mundo atraviesa por una pandemia del COVID-19; permitiendo que, con el uso de las TIC se acorten distancias, es decir, que las personas puedan comunicarse, sin la necesidad de estar presentes físicamente disminuyendo la posibilidad de contagios. Para los autores Ramos (2014); y Rueda (2012), citados por Quintero-Corzo, Munévar-Molina y Munévar-Quintero (2015): la sociedad actual ya no necesita estar presente físicamente en reuniones 0 lugares, porque ahora a través de una computadora se puede acceder a la ciber sociedad, un mundo virtual que no necesita la presencia física.

Sin embargo, el uso de las TIC, de manera continua, en el proceso educativo, durante la pandemia por COVID-19, también ha generado grandes dificultades para los educandos, como para los educadores, ya que, no se encontraban preparados ni capacitados, para el uso de las tecnologías educativas, lo cual, ha hecho que se generen espacios de exclusión para sus estudiantes, al no poder cubrir las necesidades de estos.

Otra dificultad, es el crecimiento de la brecha digital en el contexto ecuatoriano, Agudo, Pascual y Fombona (2012): definen la misma como "la diferencia existente entre países, sectores y personas tanto que tienen, como que no tienen acceso a los instrumentos y herramientas de la información y la capacidad de utilizarlos" (pág. 194). Es decir, no todos los estudiantes poseen internet estable, ni mucho menos un dispositivo electrónico para poder acceder a clases, así esta brecha digital ha producido una interrupción en el proceso de aprendizaje, limitando la adquisición y generación de conocimientos, dando paso a un bajo nivel académico o en casos extremos a una deserción escolar. 
Por lo tanto, el presente trabajo tiene como finalidad dar a conocer las aportaciones que han brindado las TIC y el conjunto de diversas aplicaciones web que la conforman, y al mismo tiempo demostrar la exclusión que se ha generado, ya sea, por su mal uso o por falta de acceso a las mismas, en el nivel de educación superior ecuatoriano, durante la pandemia por COVID-19.

\section{Desarrollo}

\subsection{Las Tics en el sistema educativo}

La pandemia por COVID-19 ha hecho que los gobiernos tomen medidas para prevenir los contagios, entre una de ellas ha sido el cierre de las instituciones educativas, para poder precautelar la salud de los estudiantes y docentes, lo cual ha generado que la educación afronte grandes retos en el trayecto de esta pandemia, teniendo que dar un giro drástico al momento de impartir clases, pasando de las clases tradicionales y magistrales en un aula física, para llevarlas a la virtualidad desde la comodidad de los hogares, en donde, tanto docentes como estudiantes tienen que buscar nuevas maneras para poder aprender, y seguir con su proceso de adquisición y generación de conocimientos, conllevando así que la mejor solución para poder continuar con el proceso educativo sea el uso de las TIC.

En este sentido, el uso de las TIC en el trayecto de esta pandemia ha permitido la continuidad del proceso educativo generando varias soluciones, entre una de ellas está la comunicación con otras personas sin la necesidad de estar presentes físicamente, por tanto, la educación superior se ha visto beneficiada, ya que, sus autoridades y docentes pueden seguir ejerciendo sus cargos y labores asignadas, mediante la modalidad de teletrabajo.

Según Quintana (2019): es una actividad que se realiza desde cualquier lugar gracias al uso de la tecnología, permitiendo que, la organización de horarios, tramites de secretaria, procesos económicos, distribución de docentes y la toma de decisiones dentro de las universidades ecuatorianas no 
Instituto Internacional de Investigación y Desarrollo Tecnológico Educativo INDTEC, C.A.

DOI: https://doi.org/10.29394/Scientific.issn.2542-2987.2021.6.22.21.397-417

OAI-PMH: http://www.indteca.com/ojs/index.php/Revista Scientific/oai

Ensayo Original / Original Essay

se vean afectadas, y por ende se pueda seguir con el proceso educativo para beneficios de los educandos.

La educación virtual ha permitido a los docentes la utilización de nuevos materiales para el proceso de aprendizaje, saliendo de la rutinaria utilización de libros físicos o de las copias, que limitaban a los estudiantes que no sean capaces de seleccionar una información distinta a la asignada por el docente. Permitiendo que, con el uso de la tecnología como lo manifiesta Moreno (2011): el uso de las TIC desarrollan habilidades de búsqueda y selección de información utilizando diferentes técnicas, ofreciendo que los educandos puedan navegar por la red, en la cual tendrán que reflexionar y seleccionar información adecuada para sus trabajos, en diferentes sitios de información como revistas, blogs, redes sociales, entre otras, logrando que el estudiante sea capaz de poder solucionar problemas, clasificar, validar información y sobre todo que genere procesos reflexivos al momento de trabajar sin el acompañamiento del docente.

Debido aquello, el estudiante pueda visualizar que su docente no es la única fuente de conocimientos, como se lo imponía en la educación presencial, sino que, a parte del profesor, se tiene una amplia gama de información en donde, educadores como educandos pueden investigar información de interés para realizar sus respectivos trabajos o tareas, logrando que el proceso de aprendizaje del estudiante sea reflexivo y que el educando sea el protagonista principal dentro del proceso educativo.

También, el uso de las TIC, como lo mencionan Flores, Aguilar, Hernández, Salazar, Pinillos y Pérez (2017): "se convierten en el puente de contacto entre el docente y el estudiante para intercambio de información que derivará en la construcción conjunta de conocimiento" (pág. 45); en otras palabras, las TIC han permitido que el educador sea un guía al momento de generar aprendizajes, y que haya un intercambio de información entre el estudiante y el docente, lo cual permitirá un proceso de aprendizaje mutuo, ya 
Instituto Internacional de Investigación y Desarrollo Tecnológico Educativo INDTEC, C.A.

DOI: https://doi.org/10.29394/Scientific.issn.2542-2987.2021.6.22.21.397-417

OAI-PMH: http://www.indteca.com/ojs/index.php/Revista Scientific/oai

Ensayo Original / Original Essay

sea con intercambio de información, opiniones o de consulta sobre diferentes temas.

Las tecnologías al permitir que el docente sea guía en el proceso de aprendizaje de sus estudiantes, también han generado que se tengan un proceso de flexibilidad al momento de impartir las clases, tal como lo expresa Álvarez (2012a):

Debido a las TIC, la educación se puede liberar de los límites del espacio, y así volverse independiente de la distancia. También se puede liberar de los límites del tiempo, por lo cual es posible que el estudiante aprenda a su propio ritmo. Los estudiantes y los profesores pueden mantener horarios y calendarios flexibles (págs. 5-6).

En este sentido, el uso de las Tics en el proceso educativo ha generado una flexibilidad en espacios como en horarios, siendo de beneficio tanto para docentes como para estudiantes, un claro ejemplo de esta flexibilidad de horarios se ha manifestado hoy en día al tener una educación virtual debido a la pandemia, en donde, algunas universidades y sus docentes han optado por dar una o dos horas clases de las tres o cuatro establecidas en los horarios, dependiendo de cada asignatura, para así dejar que los estudiantes en el tiempo restante puedan realizar un proceso de reflexión y búsqueda de información para la realización de sus tareas, evidentemente esta flexibilidad de horarios ayuda que el estudiante no se estrese y salga de ese proceso rutinario de clases, que en cierta medida no es efectivo y que solo se compone de la memorización de contenidos impartidos durante la clase.

Al tener esta flexibilidad de horarios, también se ha hecho que se respete el ritmo de aprendizaje de cada estudiante, algo que en la educación presencial no se visualizaba, ya que, se impone el momento y la forma en la que debe aprender, pero gracias a la utilización de las TIC durante esta pandemia ha permito que los docentes utilicen nuevas estrategias de aprendizaje y sobre todo dar la libertad al estudiante de cómo va hacer su tarea 
o de utilizar una fuente de información diferente a la del docente, como videos, textos, imágenes, audios, entro otros, logrando que el estudiante sea autónomo, creativo, reflexivo, y que aprenda acorde a sus necesidades, pero dejando en claro que no debe caer en la irresponsabilidad ni en el desorden.

Otro de los beneficios importantes que han generado las TIC desde su creación ha sido la posibilidad del trabajo colaborativo, tal como lo manifiesta Gros (2004), citado por Álvarez (2012b): "[...] las TIC facilitan procesos de aprendizaje individuales, así como grupales y colaborativos" (pág. 6); aunque lamentablemente no han sido aprovechadas a su máxima capacidad durante la educación presencial.

Pero durante esta pandemia, mediante la modalidad de educación virtual ha sido utilizada en mayor medida, teniendo así un trabajo colaborativoonline, que es definido por Da Silva y Ornellas (2017) como "un modelo flexible y participativo que mediante la construcción narrativa y creativa de actividades permite que niñas y niños tomen parte y sean protagonistas en actividades colaborativas mediadas por las tecnologías de la información y la comunicación (TIC)" (pág. 217); por lo tanto, esta modalidad de trabajo colaborativo-online, es una forma de poder compartir experiencias y conocimientos de manera bidireccional y continua, en la cual se puede interactuar con otros estudiantes o docentes mediante la virtualidad.

Además, el trabajo colaborativo de forma online permite a los estudiantes compartir experiencias y conocimientos, generando una comunicación bidireccional y una retroalimentación instantánea, no solo por parte del educador, sino también por parte de los compañeros de clase, logrando que se beneficien alumnos y docentes, los cuales pueden realizar actividades curriculares, gestiones o investigativas, sin la necesidad de coincidir en el mismo espacio físico.

Sin embargo, el uso de las tecnologías de la información también ha generado inconvenientes en el proceso educativo, principalmente a las 
personas que no saben utilizarla, como es el caso de los inmigrantes digitales, los que son definidos según Prensky (2010): como personas que en algún momento de su vida utilizaron las tecnologías como herramienta o por ocio, sin tener una constancia en la utilización de las mismas, lo cual hace que se tenga conocimientos nulos de cómo aplicarlas o utilizarlas en la vida diaria, ni muchos menos en el proceso de aprendizaje.

Siendo de esta manera que algunos docentes se encontraron con una gran barrera al momento de utilizar las Tecnologías de la Información y la Comunicación para dar clases, ocasionado que no sepan como captar la atención de sus alumnos o motivarlos a utilizar las herramientas digitales para su proceso de aprendizaje. Además, esta falta de conocimiento en los docentes y estudiantes sobre el uso de las Tics, también ha repercutido en que la clase no sea dinámica o atractiva, produciéndose que se tenga una clase magistral, en donde solo se habla de manera unidireccional, solo transmitiendo información y no generando aprendizajes.

Este desconocimiento sobre la utilización de las tecnologías, ha generado que las Tics no puedan cubrir con las necesidades de todos los estudiantes en relación a sus estilos de aprendizaje o al momento de brindar clases a estudiantes con algún tipo de discapacidad, ocasionando que sean apartados del grupo y en muchos casos no puedan realizar sus tareas o dar su opinión sobre algún tema.

Otra de las desventajas que han producido las Tics en la educación virtual al momento de tener un aprendizaje programado, el cual es un proceso autónomo, reflexivo y analítico, solo se califique o se vea el producto final, dejando invisibilizado el esfuerzo o el desempeño del estudiante, ya que, el docente no lo puede visualizar, como se lo hacía en la educación presencial. Siendo así que el alumnado solo se preocupe por tener un producto realizado y no por el proceso que tuvo que afrontar para lograr ese producto.

También se debe recalcar que en esta época actual se ha puesto en 
Instituto Internacional de Investigación y Desarrollo Tecnológico Educativo INDTEC, C.A.

DOI: https://doi.org/10.29394/Scientific.issn.2542-2987.2021.6.22.21.397-417

OAI-PMH: http://www.indteca.com/ojs/index.php/Revista Scientific/oai

Ensayo Original / Original Essay

evidencia la desigualdad que existen al momento de acceder a las Tics, puesto que algunos jóvenes, docentes, estudiantes con o sin discapacidad, ya sea por su condición social o economía no gozan de estas tecnologías, impidiendo su acceso a la información y por ende a su proceso educativo, y estudiantes que por lo contrario tiene acceso a dichos recursos sin ninguna dificultad.

\subsection{Aplicaciones Web}

En el mundo de las Tics, un beneficio evidente es la creación de aplicaciones en las que se pueden interactuar, crear y compartir contenido de diferente índole, tal es el caso de las redes sociales, las cuales han permitido la difusión de material educativo en sus plataformas, ya sea mediante la creación de comunidades, grupos de aprendizaje o de forma individual, ocasionado que las personas o estudiantes al momento de estar conectadas a una red social, (Facebook, Instagram, WhatsApp, entre otras) puedan informase o encontrar en ciertos casos información de forma rápida y veras, siendo de esta manera que las plataformas de redes sociales no solo sean vistas como espacios de ocio, sino también como espacios que fomenten la educación, en donde se puede tener una comunicación bidireccional por medio de comentarios o incluso de contactarse con el propietario del contenido.

Para Valenzuela (2013); Muñoz, Fragueiro y Ayuso (2013): las redes sociales al ser aplicaciones que cada día crecen y aparecen más de ellas en el mundo, han traído grandes beneficios para el área educativa, tal es el caso que, las redes sociales proporcionan un amplio acceso a los recursos de información, reducen las barreras a la interacción de grupo y la comunicación.

En concordancia con los autores, las redes sociales como Facebook, han permitido que se rompan barreras de comunicación entre docentes, estudiantes y personas en general, ya que, por medio de esta aplicación se puede interactuar de manera directa con la otra persona, ya sea mediante un comentario en su publicación, o por los conocidos emojis, en donde se puede 
Instituto Internacional de Investigación y Desarrollo Tecnológico Educativo INDTEC, C.A.

DOI: https://doi.org/10.29394/Scientific.issn.2542-2987.2021.6.22.21.397-417

OAI-PMH: http://www.indteca.com/ojs/index.php/Revista Scientific/oai

Ensayo Original / Original Essay

demostrar si la información publicada ha sido o no de interés para el usuario que lo busca, algo que sin duda al estar mediante una interacción física no se da, ya sea por temor, vergüenza o por no poder expresar su ideas delante de otras personas.

Durante esta pandemia las redes sociales han proporcionado ayuda a la educación, facilitando la comunicación y búsqueda de material didáctico o informativo para estudiantes y docentes, a través de los miles de páginas educativas que existen en Facebook o Instagram. Además, han permitido que los estudiantes y docentes puedan desarrollar su imaginación, creando videos, imágenes e infografías que sintetizan la información, siendo estas más atractivas y entendibles para el alumno o cualquier otra persona interesada en el tema.

Otro punto de beneficio que ha generado las redes sociales es la oportunidad de trabajar de forma colaborativa, como por ejemplo en la aplicación WhatsApp, en donde se pueden crear grupos educativos para compartir imágenes, audios, textos, videos o documentos, acorde a la necesidad o tema establecido en dicho grupo, permitiendo así que los docentes y alumnos puedan aprender uno del otro, compartiendo y debatiendo sus conocimientos, dejando atrás esa visión que el docente es la única fuente de conocimientos.

Además, al crear estos grupos con fines educativos, los estudiantes están al pendiente de sus tareas o temas por aprender, debido a que los jóvenes pasan su mayor parte de tiempo en las redes sociales, generando que, al tener un grupo o espacio educativo dentro de ellas, sea más atractivo aprender, consultar y por ende no olvidarse de sus compromisos educativos.

Las redes sociales han proporcionado varias ventajas para la educación, sin embargo, se debe mencionar algunas desventajas que han generado para el sistema educativo, tal como lo muestra Marco y Chóliz (2013): 


\section{Ensayo Original / Original Essay}

- Genera distracciones en otras actividades.

- Es incompatible con otras actividades, o resta tiempo.

- Provoca dependencia.

La constante utilización de las redes sociales durante esta pandemia ha provocado que los estudiantes estén más tiempo emergidos en las mismas, provocando la necesidad una respuesta inmediata sobre algún tema, lo cual representa tener un estrés y frustración por no poder conseguir la misma, además, al tener esta dependencia, ocasiona que los estudiantes tengan menos entusiasmo al momento de investigar y poder seleccionar información veraz.

Otra de las desventajas que han provocado las redes sociales al ser utilizadas en la educación durante esta pandemia, es la exclusión del alumnado que tiene algún tipo de discapacidad ya sea visual o auditiva, puesto que, la interfaz de estas aplicaciones no son totalmente accesibles, por ejemplo; para una persona con discapacidad visual le resulta complejo poder acceder a todas las funcionalidades de Facebook, debido a que su interfaz posee demasiados accesos o funciones distribuidas en diferentes partes de la pantalla, lo que ocasiona que un estudiante con discapacidad visual no tenga el mismo acceso y oportunidad que los demás educandos al momento de realizar un trabajo o realizar una búsqueda de información, lo cual genera una desmotivación en el alumno.

De igual manera la aplicación WhatsApp también presenta desventajas al ser utilizada por alumnos con discapacidad auditiva, ya que, al momento de enviar audios o videos sin subtítulos, el estudiante no puede comprender lo que se trata en ellos

Esta aplicación no genera la transformación de audios a textos lo que dificulta que el alumno no pueda entender ordenes emitidas por sus docentes, 
o cuando sus compañeros utilizan audios, no permite que el alumno con discapacidad auditiva pueda saber lo que se habla o se comparte en los grupos de WhatsApp, siendo esta una dificultad que genera exclusión en el sistema educativo, limitando la capacidad del alumno al momento de aprender y por ende tener vacíos en su proceso de aprendizaje.

También se debe recalcar que, una de las dificultades más grandes que se encuentra al momento de utilizar estas redes sociales en el sistema educativo es la falta de dispositivos electrónicos y de conexión a una red estable de internet, lo que genera una barrera al momento de brindar una educación de calidad, algo que sin duda es evidente en esta educación virtual, en la cual, varios alumnos no han podido acceder a sus clases universitarias, debido a estos inconvenientes.

El uso de las Tics en la educación ha permitido incorporar herramientas multimedia interactivas, las cuales, según Enríquez (2020a): “que favorezcan al desarrollo de habilidades tales como, de percepción, de lenguaje, cognitivas, sociales, emocionales y visuales" (pág. 874); siendo una de estas herramientas la más conocida y utilizada PowerPoint al momento de crear contenido para presentar o informar sobre temas educativos.

Sin embargo, para Enríquez (2020b): hoy en día existen aplicaciones o herramienta que permiten realizar presentaciones más interactivas acorde a las necesidades del alumnado un claro ejemplo es Genially, la misma es una herramienta web que ofrece realizar presentaciones de manera interactiva, permitiendo utilizar diversas funciones en planes gratuitos, mientras que las funcionalidades avanzadas se las puede poseer mediante un plan de pago.

La aplicación Genially, en la actualidad ha tenido un gran impacto en la educación, ya que, ha permitido a los docentes y alumnos realizar un sin fin de presentaciones más atractivas que las de su gran rival PowerPoint, captando la atención de las personas que lo visualizan, debido a que permite agregar efectos, hipervínculos, símbolos, formas, videos, enlaces de 
diferentes páginas, entre otros, las cuales pueden ser personalizadas según el requerimiento del usuario.

Genially permite las creaciones de diferentes recursos educativos, como presentaciones, juegos, imágenes interactivas, infografías, libros interactivos, videos, murales, mapas conceptuales, guías, entre otros, que ayudan a reducir las explicaciones magistrales, puesto que toda la información teórica se encuentra plasmada en las diferentes capas o páginas de Genially, permitiendo que el alumno pueda descubrirlo de forma intuitiva, que conforme a González (2019a): genera que los estudiantes tengan curiosidad sobre el tema a tratar, asimismo, esta aplicación permite que el estudiante pueda desarrollar su autonomía.

Al permitir Genially el uso de imágenes, iconos, mapas, elementos interactivos, formularios, quizzes e incluso archivos de otras fuentes, según González (2019b): permiten "la creación de un entorno de aprendizaje similar a la vida real que ayuda a contextualizar la lección, maximizar la memorización, el pensamiento crítico y la creatividad, creando metáforas visuales" (pág. 5); lo cual es un punto esencial, puesto que, con ello se puede trabajar con la realidad que estamos atravesando; la pandemia por COVID-19, y de esta manera poder establecer una relación entre docentes y alumnos, sin perder la empatía emocional, ni la habilidad de socialización entre ellos.

El uso de la plataforma o aplicación Genially, ha generado que se pueda atender a la gran mayoría de los aprendizajes que posee el alumnado, ya que, por medio de las imágenes, videos y audios, se puede captar la atención de los estilos de aprendizaje visual y auditivo al mismo tiempo, mientras que, con la insertación de texto se pueda trabajar el aprendizaje verbal o la parte teórica que para algunos alumnos es esencial.

No solo otorga que el objeto o contenido cobre vida, sino que también, permite enfocarse en los contenidos fundamentales, permitiendo tener una secuencia lógica de ideas principales y secundarias esenciales al momento de 
recordar o revisar el contenido impartido.

Otra de las ventajas que proporciona Genially a la educación virtual, es la gamificación, la misma que ha sido poco utilizada. Debido a la falta de creatividad y conocimientos sobre aplicaciones que generen el juego en el trayecto de la modalidad virtual. Es por ello que Genially cuenta con una gran cantidad de plantillas prediseñadas que fomentan el aprendizaje a través del juego, coincidiendo con González (2019c): este tiene "una mayor involucración del alumnado y, por lo tanto, se maximiza el recuerdo de los contenidos. Al emocionar al alumnado, el aprendizaje fluye" (pág. 6).

Sin embargo, el uso de Genially también ha presentado desventajas al momento de utilizarla en la educación virtual, entre ellas la inaccesibilidad para las personas con discapacidad visual, ya que su interfaz no es compatible con los diferentes lectores de texto, pues sus herramientas se encuentran distribuidas en diversas partes de la pantalla, lo que impide su utilización, generando que las personas con discapacidad visual no tengan la oportunidad de crear o consumir contenido en esta plataforma, produciendo una barrera al momento de utilizarla en las diferentes aulas de clase tanto, en la presencialidad, como en la virtualidad.

Otra de las desventajas que presenta Genially al momento de ser utilizada en dispositivos electrónicos móviles, conforme a Enríquez (2020c): es que su interfaz "no es bueno ya que requiere una gran amplitud para visualizarse correctamente" (pág. 882); generando de esta manera un impedimento para las personas con o sin discapacidad crear contenido desde su celular, lo que provoca un inconveniente, puesto que no todos cuentan con una computadora. También, se debe recalcar, que esta plataforma o aplicación no es offline, debido a que necesita una conexión estable a internet para su utilización.

El empleo de las Tics y el conjunto de aplicaciones que la conforman, han generado soluciones a problémicas que se han presentado en la 
educación virtual, debido a la pandemia por COVID-19, tal es el caso que ha permito a los docentes, autoridades educativas, y estudiantes continuar con el proceso educativo. Pero se debe recalcar que, el uso de estas ha generado inconvenientes, tanto en los educandos como en los educadores, ya sea por la falta de conexión a internet, falta de dispositivos electrónicos PC, celulares o tabletas, o por el simple hecho de no saber cómo utilizarlas.

Es por ello que estas tecnologías deben ser exploradas y aprovechadas al máximo, especialmente las que son de uso offline como es Word, Excel o PowerPoint, ya que las mismas permiten realizar trabajos sin la necesidad de tener conexión a internet o poseer un dispositivo de alta gama, siendo estas y otras aplicaciones en línea esenciales al momento de trabajar en la educación virtual o a distancia.

\section{Conclusiones}

El uso de las tecnologías durante esta educación virtual, debido a la pandemia por COVID-19, ha permitido que los docentes y estudiantes puedan seguir con su proceso de enseñanza aprendizaje, dando acceso a la información desde cualquier lugar o contexto, pero, se debe recalcar que, la brecha digital existente en diversos contextos, ya sea por el nivel social, etnográfico o económico, ha repercutido que no todas las personas tengan las mismas oportunidades para acceder y utilizar las tecnologías, conllevando que se puedan producir casos de exclusión en el sistema educativo.

Siendo de esta manera que, se debe considerar a las tecnologías como un acceso fundamental, esencial y universal para la vida del ser humano, ya que, las mismas proporcionan una gran variedad de ventajas, como la comunicación con otras personas sin la necesidad de estar presentes físicamente, lo cual, ha sido un beneficio en la actualidad para frenar los casos por COVID-19, permitiendo el acceso a miles de fuentes de información veraz, de manera más rápida y eficaz. 
Asimismo, el uso de las TIC y su gran conjunto de aplicaciones que lo conforman deben ser puestas en práctica bajo una metodología o con un objetivo previamente diseñado, para que no se produzcan desigualdades 0 casos de exclusión para alumnos y docentes que posean algún tipo de discapacidad o falta de acceso a las mismas.

También, se debe considerar que para la correcta utilización de las tecnologías se debe realizar una difusión o capacitación a los docentes que no poseen habilidades en las mismas, para que puedan cubrir las necesidades de todos los estudiantes, y generen un aprendizaje continuo y bidireccional, permitiendo a los alumnos desarrollar la imaginación, autonomía, creatividad y ser generadores de conocimientos, y dejar de ser simples receptores de información.

Por último, se debe hacer énfasis que el uso de las redes sociales en la educación debe ser utilizadas de forma conjunta, con diferentes materiales o aplicaciones, para que así se tengan las mismas oportunidades de participación por parte de todos los alumnos. Las redes sociales deben ser empeladas como un complemento o ayuda al momento de impartir conocimientos, y no como una estrategia continua y agobiante, para que, el estudiante no tenga miedo de utilizarlas ni de rechazarlas en su vida diaria y sobre todo el docente debe tener en cuenta las necesidades de sus alumnos y estar inmerso en la utilidad de estas tecnologías, para que no se produzcan casos de desmotivación o exclusión educativa.

\section{Referencias}

Agudo, S., Pascual, M., Fombona, J. (2012). Usos de las herramientas digitales entre las personas mayores. Comunicar, (39), 193-201, eISSN: 1134-3478. Recuperado de: https://doi.org/10.3916/C39-2012$\underline{03-10}$

Álvarez, G. (2012a,b). Las nuevas tecnologías en el contexto universitario: 
sobre el uso de blogs para desarrollar las habilidades de lectoescritura de los estudiantes. Revista de Universidad y Sociedad del Conocimiento (RUSC), 9(2), 3-17, e-ISSN: 1698-580X. Recuperado de: http://dx.doi.org/10.7238/rusc.v9i2.1160

Da Silva, M., \& Ornellas, A. (2017). Potencialidades de un modelo colaborativo para la apropiación de las TIC en un contexto de vulnerabilidad social. Revista Psicopedagogía, 34(104), 216-227, eISSN: 0103-8486. Brasil: Associação Brasileira de Psicopedagogía.

Enríquez, M. (2020a,b,c). Características de las herramientas multimedia para el desarrollo de Presentaciones Interactivas. Journal of Science and Research, 5, 873-891. Recuperado de: https://revistas.utb.edu.ec/index.php/sr/article/view/1044

Flores, M., Aguilar, A., Hernández, Y., Salazar, J., Pinillos, J., \& Pérez, C. (2017). Sociedad del conocimiento, las TIC y su influencia en la educación. Revista Espacios, 38(35), 39-50, e-ISSN: 0798-1015. Recuperado de:

https://www.revistaespacios.com/a17v38n35/a17v38n35p39.pdf

González, M. (2019a,b,c). Genially. Libros interactivos geniales. ISSN: 2695-4176. Madrid, España: Ministerio de Educación y Formación Profesional; Instituto Nacional de Tecnologías Educativas y de Formación del Profesorado (INTEF).

Marco, C., \& Chóliz, M. (2013). Tratamiento cognitivo-conductual en un caso de adicción a Internet y videojuegos. International Journal of Psychology and Psychological Therapy, 13(1), 125-141, e-ISSN: 15777057. Recuperado de:

https://www.redalyc. org/articulo.oa?id=56025664009

Martínez, O. (2018). Uso de las Tecnologías de la Información y la Comunicación en la Educación Básica. Revista Scientific, 3(10), 154174, e-ISSN: 2542-2987. Recuperado de: 
https://doi.org/10.29394/Scientific.issn.2542-2987.2018.3.10.8.154-174 MINEDUC (2020). Acuerdo Nro. MINEDUC-MINEDUC-2020-00020-A. Ecuador: Ministerio de Educación.

Moreno, M. (2011). Conocimiento y Uso de las TIC desde la perspectiva de los estudiantes de la Universidad de Sonora. Tesis. México: Universidad de Sonora.

Muñoz, M., Fragueiro, M., \& Ayuso, M. (2013). La importancia de las redes sociales en el ámbito educativo. Escuela Abierta, 16, 91-104, e-ISSN: 1138-6908. Recuperado de:

https://ea.ceuandalucia.es/index.php/EA/article/view/159

OMS (2020). La OMS caracteriza a COVID-19 como una pandemia. Ginebra, Suiza: Organización Panamericana de la Salud; Organización Mundial de la Salud.

Prensky, M. (2010). Nativos e Inmigrantes Digitales. Depósito legal: M24433-2010. España: Edita Distribuidora SEK, S.A.

Quintana, J. (2019). Estudio de avance en la implementación del teletrabajo en la Universidad Nacional Abierta y a Distancia UNAD 2016 - 2019. Monografía. Bogotá, Colombia: Universidad Nacional Abierta y a Distancia UNAD. Recuperado de:

https://repository.unad.edu.co/handle/10596/31117

Quintero-Corzo, J., Munévar-Molina, R., \& Munévar-Quintero, F. (2015).

Nuevas tecnologías, nuevas enfermedades en los entornos educativos. Revista Hacia la promoción de la salud, 20(2), 13-26, eISSN: 0121-7577. Recuperado de:

https://www.redalyc.org/articulo.oa?id=309143500002

Valenzuela, R. (2013). Las redes sociales y su aplicación en la educación.

Revista Digital Universitaria, 14(4), 1-14, e-ISSN: 1067-6079. Recuperado de:

http://www.revista.unam.mx/vol.14/num4/art36/index.html 


\section{Ensayo Original / Original Essay}

\section{Manuel Esteban Rojas Bustos}

e-mail: esteban.rojasb4875@hotmail.com

Nacido en Azogues, Ecuador, el 14 de julio del año 1995.

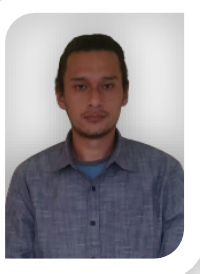

Realicé mis estudios de segundo nivel en la Unidad Educativa "Luis Rogerio González", en donde obtuve el título de Bachiller Técnico en Electrónica de Consumo; en mi proceso de formación como futuro docente, formo parte del grupo de investigación GIET, en donde he tenido la oportunidad de poder participar como ponente en congresos internacionales tales como el "I Coloquio Internacional Universitario: Educación Inclusiva con Enfoque de Género en la COVID-19" en el año 2021; también desempeño la función de comunicación y difusión de información en las redes sociales en dicho grupo; se debe recalcar que me interesan los temas relacionados con la inclusión, calidad educativa y sobre todo emprendimientos, ya que es esencial para la posterioridad. 


\section{Ensayo Original / Original Essay}

\section{Lissbeth Johanna Aucancela Coraizaca e-mail: liss.joha05@gmail.com}

Nacida en Azogues, Ecuador, el 15 de octubre del año

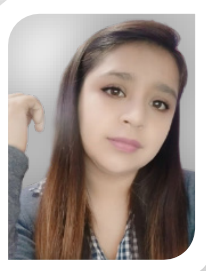
1999. Realicé mis estudios de segundo nivel en la Unidad Educativa "Luis Rogerio González", en donde obtuve el título de Bachiller Técnico Polivalente en Contabilidad y Administración; en mi proceso de formación académica, formo parte del grupo de investigación GIET en el cual he podido realizar diferentes investigaciones con temática relacionada a la educación, inclusión e innovación, dentro del grupo he participado en simposios y coloquios internacionales, tales como el $1 \mathrm{er}$ Coloquio Internacional Universitario: Educación Inclusiva con Enfoque de Género en la COVID-19 en el año 2021; también debo mencionar que pertenezco al grupo Coworking Inclusivo.

El contenido de este manuscrito se difunde bajo una Licencia de Creative Commons ReconocimientoNoComercial-Compartirlgual 4.0 Internacional 